\title{
The direct measurement of embryogenic volume and nucleo-cytoplasmic ratio during mouse pre-implantation development
}

\author{
Catherine E M Aiken, Peter P L Swoboda, Jeremy N Skepper and Martin H Johnson \\ Department of Anatomy, Downing Street, Cambridge CB2 3DY, UK \\ Correspondence should be addressed to M H Johnson; Email: mhj@mole.bio.cam.ac.uk
}

\begin{abstract}
After fertilization, the mammalian conceptus undergoes cleavage, a process of cell proliferation in the absence of interphase growth. It is not known when cleavage ends and gives way to fully replicative cell cycles with a stable nucleo-cytoplasmic ratio. We have used two-photon excitation and confocal microscopy to measure directly volumes and nucleo-cytoplasmic ratios of whole murine concepti and their individual constituent blastomeres during pre-implantation development up to the early uterine attachment stage (day 5). We show that the total cytoplasmic volume of the conceptus remains constant during pre-implantation development, and that the average nucleo-cytoplasmic ratio increases exponentially throughout the same period. Data from individual blastomeres show that both volume and nucleo-cytoplasmic ratio diverge in the inner and outer subpopulations evident from the 16-cell stage (fifth developmental cycle) onwards. Cells from emergent outer trophoblast populations are larger and have smaller nucleo-cytoplasmic ratios than those from emergent inner pluriblast populations. Moreover, the nucleo-cytoplasmic ratio of the trophoblast appears to be stabilizing, suggesting that for this subpopulation cleavage may end at the 16-32-cell transition. Putative hypoblast and epiblast cell subpopulations within the pluriblast were not distinguishable by volume or nucleo-cytoplasmic ratio. Embryonic stem cell volume was higher than that of either cell subpopulation of expanded blastocysts, and their nucleo-cytoplasmic ratio was similar to that of trophoblast cells.

Reproduction (2004) 128 527-535
\end{abstract}

\section{Introduction}

Most proliferative cell cycles involve cell growth and chromosomal replication during interphase followed by division to smaller daughter cells during M-phase, such that both cell numbers and total cell mass increase (O'Farrell 2004). Recent work has explored how growth is coupled to cell cycle progression so as to control the size of the maternal cell and thereby its mitotic offspring (Kellogg 2003, Fingar et al. 2004). However, some mitotic cells do not show interphase growth, for example the period of post-fertilization development in many animal species, traditionally called 'cleavage' cell cycles. Cleavage is a process by which a large oocyte, laden with developmental information and energy supplies, is converted to a multi-cellular embryo or larva. It is not known how growth is suppressed during cleavage. Cleaving cells in some organisms are characterized by absence of the $\mathrm{G}_{1}$ and $G_{2}$ phases, and it has been suggested that this feature is important as it offers no opportunities for growth checkpoints to function. However, during mouse cleavage, although the $\mathrm{S}$ phase does occupy most of each of the first five developmental cell cycles, G phases are present, even though short and variable, at least some cdk:cyclin oscillations occur, and there is evidence for some checkpoint activity (Streffer et al. 1980, Howlett et al. 1985, Smith \& Johnson 1986, Chisholm 1988, Moore et al. 1996, Fulka et al. 1999, Bemark et al. 2000, Takai et al. 2000, Waclaw \& Chatot 2004). Cleavage in the mouse also differs from other organisms in that the oocyte is smaller than many others (60-100 $\mu \mathrm{m}$ diameter), early cleavage cell cycles are longer $(12-24 \mathrm{~h})$, transcriptional activation occurs soon after fertilization (Telford et al. 1990, Schultz 2002), development occurs within the nutritive female genital tract, and early development is primarily concerned with the elaboration of a system of membranes for tapping maternal resources (Johnson \& Selwood 1996, Johnson \& Day 2000, Johnson \& McConnell 2004).

In this paper, we derive quantitative data on cleavage for the pre-implantation period of development. We describe the direct measurement of total conceptus volume and nucleo-cytoplasmic ratio and examine 
whether the emergent cell lineages differ in cell volume and nucleo-cytoplasmic ratio.

\section{Materials and Methods}

\section{Recovery and handling of eggs and concepti}

Female CD1 and MF1 mice (Harlan, Bicester, UK) of 3-6 weeks were housed in groups of between five and ten. Procedures were carried out under Home Office license and after local ethical review. Ovulation was induced by intraperitoneal injection of PMS (10 iu; Folligon; Sigma) followed after $46-48 \mathrm{~h}$ by intraperitoneal injection of human chorionic gonadotropin (10 iu; Chorulon; Sigma). Females were paired with individually housed CD1 stud males (Harlan) and mating assessed by inspecting for a vaginal plug. Concepti were retrieved from the oviducts or uteri between day 0 (day of positive vaginal plug) and early day 5 (when attachment is initiated) by flushing with $\mathrm{M} 2+4 \%$ (w/v) BSA culture medium (M7167 embryo tested; Sigma) and stored until use under oil (M8410 embryo tested; Sigma). Recovery and transport were at $37^{\circ} \mathrm{C}$. All concepti were imaged live. When removal of the zona was required, brief exposure to acid Tyrode's solution (made in the laboratory) was used. Intact inner cell masses (ICMs) were recovered from blastocysts by immunosurgery (Solter \& Knowles 1975). Concepti were recovered from uteri $98 \mathrm{~h}$ post-human chorionic gonadotropin and sorted into expanded, semi-expanded, nascent and pre-nascent blastocysts. Zona-intact blastocysts were incubated for $30 \mathrm{~min}$ in a $1: 4$ dilution of rabbit antitrophoblast antibody (a gift from Azim Surani, Department of Physiology, University of Cambridge, Downing Street, Cambridge, UK), washed through four changes of $\mathrm{M} 2+4 \%$ BSA, and incubated in a $1 / 5$ dilution of guineapig complement (Sigma) for $20 \mathrm{~min}$. The blastocysts were then transferred to $\mathrm{M} 2+4 \% \mathrm{BSA}$ for $30 \mathrm{~min}$, and the ICMs removed by gentle pipetting through a micropipette. Disaggregation of concepti was achieved by one of two methods. Up to the 16-cell stage, zona-free concepti were exposed for 10-30 min to calcium-free M2+6\% BSA (made in the laboratory). From the 16-cell stage onwards, zona-free concepti were exposed to $0.1 \%$ trypsin (containing $0.04 \%$ EDTA; Handyside 1980) in calcium-free $\mathrm{M} 2+4 \%$ BSA. Embryonic stem (ES) cells (CES3 on 129/Sv background; a gift from Azim Surani) were stained and imaged, and underwent volumetric analysis using the same techniques as for concepti.

\section{Imaging concepti}

Nuclei were stained by incubating concepti in $\mathrm{M} 2+4 \%$ BSA containing $10 \mu \mathrm{g} / \mathrm{ml}$ Hoechst 33258 (Sigma; $1 \mathrm{mg} / \mathrm{ml}$ stock) for $2-4 \mathrm{~h}$. Cytoplasm was stained by a $15 \mathrm{~min}$ incubation in the calcium dye Calcein AM $(0.5 \mu \mathrm{g} / \mathrm{ml}$ in $\mathrm{M} 2+4 \%$ BSA; Molecular Probes, Eugene, ON, USA). Membranes were stained with the styryl dye FM4-64 $(3.025 \mu \mathrm{g} / \mathrm{ml}$; Molecular Probes), which was added to the viewing chamber. In the majority of experiments, triple staining was used. Hoechst-stained concepti were rinsed thoroughly in protein-free PBI (phosphate buffered medium 1 ; supplied courtesy of Sheila Barton, Department of Physiology, University of Cambridge, Downing Street, Cambridge, UK) and placed in $1 \mathrm{ml}$ of $\mathrm{PBI}$ in the centre of a $30 \mathrm{~mm}$ petri dish to the base of which had been fixed a No. 1 coverslip using Sylgard (Dow Corning). The coverslips were pre-coated with either poly-L-lysine $(1 \%$ in PBS) or (mostly) phytohaemagglutinin $(0.1-0.2 \mathrm{mg} / \mathrm{ml}$; Sigma) followed by washing with PBI. After concepti had firmly adhered to the coverslip, $1 \mathrm{ml} \mathrm{M} 2+4 \%$ BSA was added. Whole concepti were imaged with their zonae intact at $37^{\circ} \mathrm{C}$ using a Leica TCS-SP-MP two-photon excitation and confocal microscope. Visualization was by sequential two-photon excitation and confocal microscopy, using a picosecond pulsed tunable Tsunami laser (Spectra Physics, Mountain View, CA, USA), tuned to $775 \mathrm{~nm}$ to excite Hoechst and Calcein and the $568 \mathrm{~nm}$ line of a Krypton laser to excite FM4-64. A water immersion $\times 63$ lens with a numerical aperture of 1.2 and a cover-glass correction collar were used. Emitted fluorescent light was captured in discrete windows (Hoechst 400-480 nm, FM4-64 600-700 nm, and Calcein 505$545 \mathrm{~nm}$ ). The confocal pinhole was set to 1 Airy disk equivalent for the objective lens in both confocal and twophoton imaging. A Z-series of images was collected from each subject, capturing an image every $1 \mu \mathrm{m}$. Every image was used for three-dimensional (3D) reconstruction, while every second image was used for stereology.

\section{Analytical procedures}

The Cavalieri principle (Gunderson \& Jenson 1987) was used to estimate volumes of concepti, ICMs, single cells and their nucleo-cytoplasmic components. Images on screen $(521 \times 521 \mathrm{~mm})$ were overlain with quadratic grids with a spacing of $20 \mathrm{~mm}$ for cytoplasm and $10 \mathrm{~mm}$ for nuclei. The number of intersections overlying nuclei and cytoplasm was counted. The near-spherical shape of the concepti meant that less than the accepted maximum of 200 points per object from 10-15 sections could be counted, giving an error coefficient of $5-10 \%$ (Roberts et al. 1993, 1994). The volume of the conceptus (excluding blastocoelic volume) or its constituent cytoplasm or nuclei could then be calculated. For single-cell analysis, Z-stacks were captured through the conceptus and individual cells within these images were chosen for analysis. Where cell subpopulations were analysed, random selection of cells was attempted. However, not all cells within the total image were delineated clearly enough to obtain an accurate volume. Data from these cells are not included, and so a degree of non-random selection cannot be excluded. The nucleo-cytoplasmic ratio was also calculated for isolated single cells, although these are not comparable directly to those obtained through whole conceptus analysis due to problems with different levels 


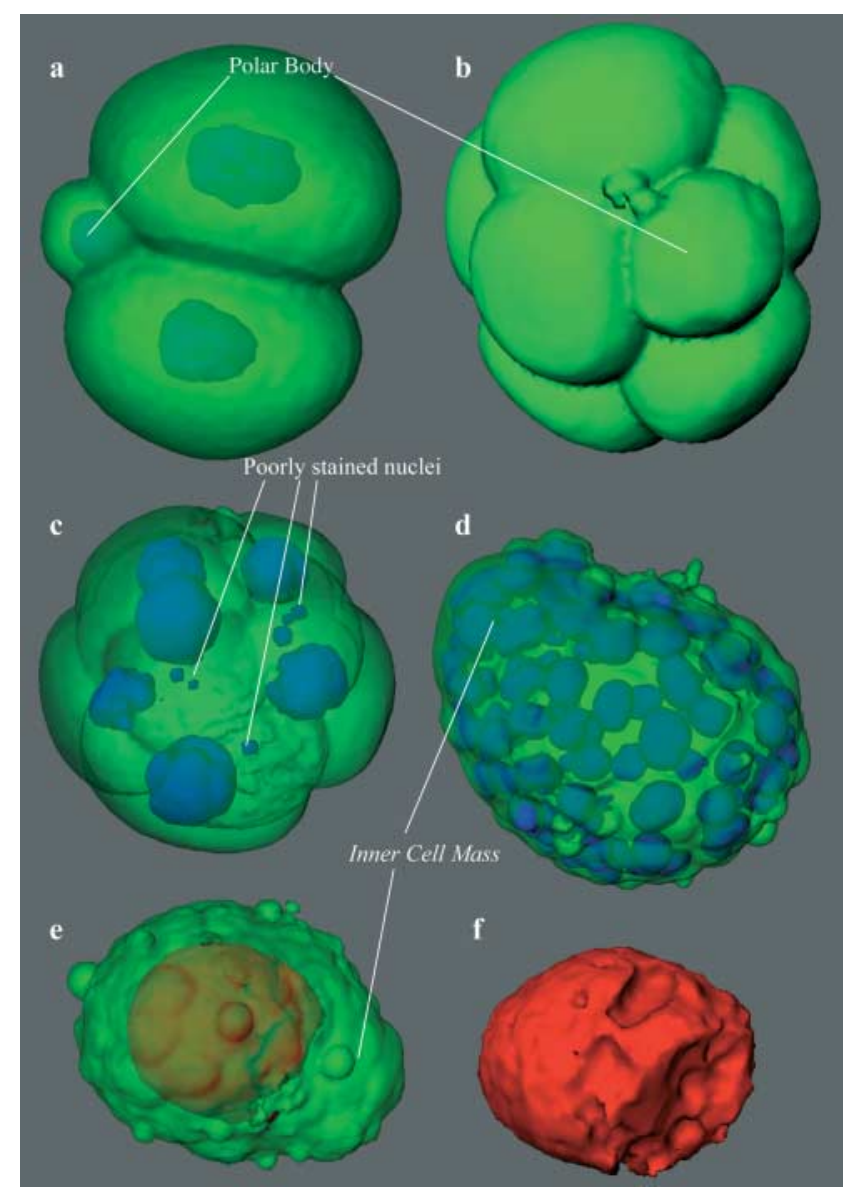

Figure 1 Examples of the 3D models made using Imaris v.3 software (BitPlane AG) and used for volume analysis. Nuclei are shown in blue, cytoplasm in green and blastocoele in red. (a) Two-cell stage; (b) solid model of an eight-cell stage; (c) same eight-cell stage as in (b) but rotated and with the cytoplasm appearing translucent to show nuclei; (d) expanded blastocyst; (e) same blastocyst as in (d), without nuclei but showing blastocoele created by inserting the cytoplasmic image and increasing the acceptance threshold; (f) blastocoele from (e).

of nuclear staining and filter corrections. Independent estimates of total conceptus volume were also made on 3D reconstructions (Fig. 1) made from sectional data using Imaris v.3 software (BitPlane AG, Zurich, Switzerland).
Gamma correction and a median filter were applied to sectional images to achieve a satisfactory 3D image. Volume estimates of the same concepti made using the Cavalieri method and 3D reconstructions did not differ significantly $(P<0.0001)$. 3D reconstruction using Imaris proved impossible to use reliably for calculation of the nucleo-cytoplasmic ratio, for technical reasons. Thus, the degree of variability in staining between individual nuclei prevented reconstruction adequate for measurement purposes (see Fig. 1c).

\section{Statistical tests}

Data from whole conceptus analysis have been plotted such that each data point represents a single conceptus $(n=86)$. Pearson correlation coefficients were calculated, and are included in the figure legends. For single-cell analysis, cells from concepti of each developmental stage were grouped together and the mean $\pm 95 \%$ confidence interval plotted. Sample sizes ( $n$ values) are given in Table 1 and the figure legends.

\section{Results}

\section{Total conceptus volume and averaged nucleo- cytoplasmic ratio over the pre-implantation period}

Examples of concepti used for analysis are shown in Fig. 2. The total volume of concepti (excluding blastocoelic volume) increases slightly during the pre-implantation period, and can be accounted for entirely by the increasing total nuclear volume with each successive cell cycle (Fig. 3a), while whole embryo cytoplasmic volume remains constant. Mean cytoplasmic volume per cell was calculated by division from whole conceptus data and found to decrease exponentially throughout the preimplantation period (Fig. $3 b$ and $c$ ), consistent with cleavage divisions continuing throughout this period. The data from Fig. $3 a$ were also used to calculate the changing mean nucleo-cytoplasmic ratio, which was found to increase during pre-implantation development (Fig 3d). Together with data showing that cytoplasmic volume remains constant (Fig 3a), these results imply that within

Table 1 Volumetric analysis of single blastomeres from mouse concepti during the first seven developmental cycles.

\begin{tabular}{|c|c|c|c|c|}
\hline Developmental cell cycle & Total cell number per conceptus & Mean cell volume $\left(\mu \mathrm{m}^{3}\right)$ & Confidence limits & Number of cells counted \\
\hline $1 \mathrm{st}$ & 1 & 185558.67 & 17079.76 & 20 \\
\hline $2 \mathrm{nd}$ & 2 & 115192.43 & 4769.14 & 26 \\
\hline $3 \mathrm{rd}$ & 4 & 48081.23 & 1961.63 & 40 \\
\hline 4 th & 8 & 21665.50 & 1997.57 & 40 \\
\hline 5th & 16 inner & 6442.13 & 496.37 & 14 \\
\hline 5 th & 16 outer & 11569.68 & 1322.79 & 40 \\
\hline 5 th-6th & 28 inner & 3814.08 & 315.39 & 40 \\
\hline 5 th-6th & 28 outer & 5504.62 & 386.80 & 40 \\
\hline 6th & 32 inner & 2039.99 & 185.33 & 92 \\
\hline 6th & 32 outer & 3399.76 & 584.42 & 44 \\
\hline 7th & 64 inner & 517.93 & 50.62 & 35 \\
\hline 7 th & 64 outer & 1092.84 & 140.08 & 37 \\
\hline
\end{tabular}



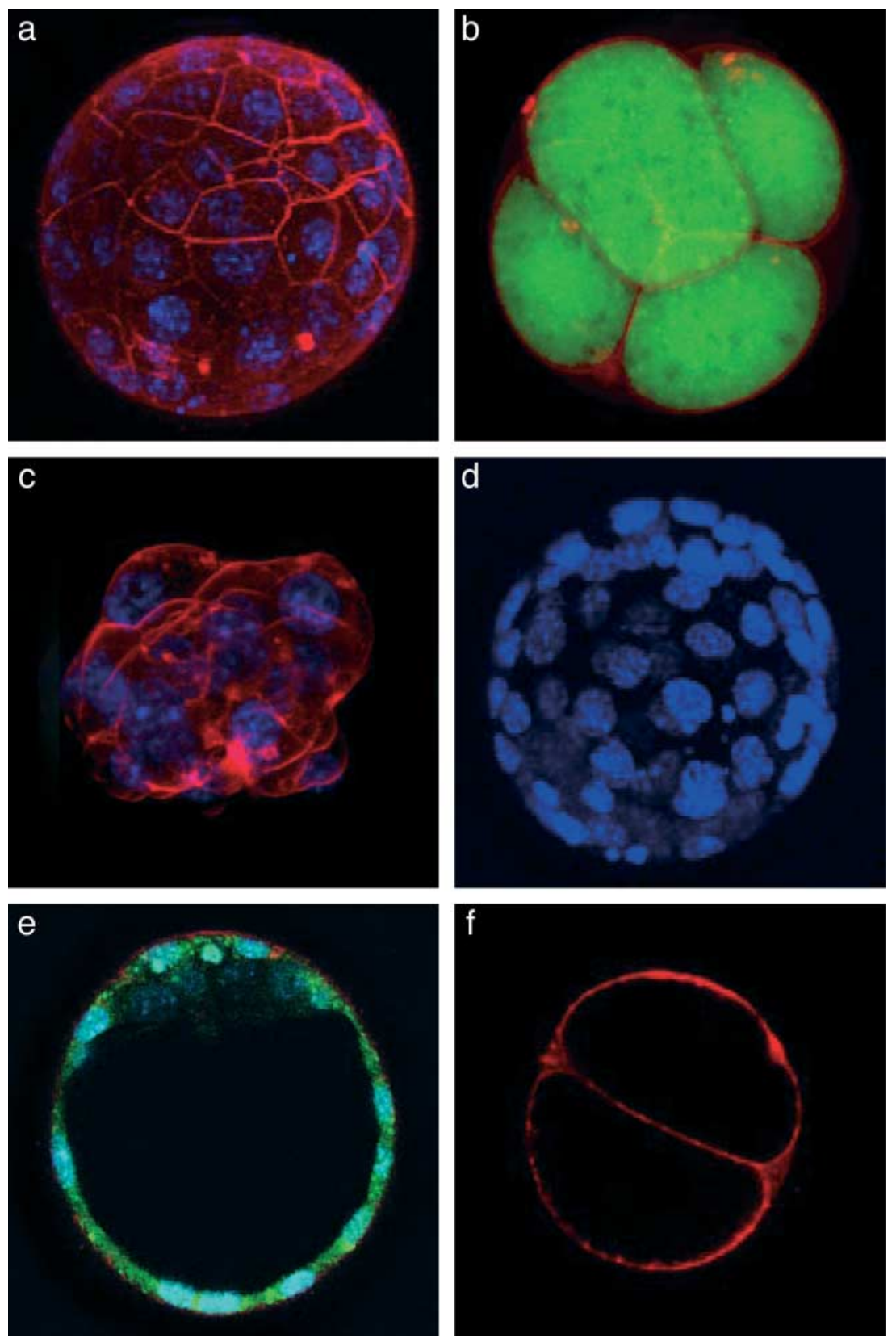

Figure 2 Examples of projected images of sections $(a-d)$ and of optical sections used for Cavalieri analysis $(e, f)$. Cell membranes are shown in red, cytoplasm in green and nuclei in blue. Actual image sizes are $512 \times 512 \mu \mathrm{m}$. (a) Projection of optical sections of 3.5-day blastocyst shows overlaid cell membranes and nuclei. (b) Projection of four-cell stage shows overlaid cytoplasm and cell membranes. (c) Projection of isolated ICM shows overlaid cell membranes and nuclei. (d) Projection of nuclei of 3.5-blastocyst; images of projected nuclei were used to determine cell number per conceptus. (e) Optical section through 3.5-day blastocyst shows overlaid cell membranes (only outer membrane stained in this preparation), cytoplasm and nuclei. (f) Optical section through two-cell stage shows cell membranes. the conceptus as a whole, interphase growth has not occurred and that cleavage divisions have not terminated by the time of attachment.

The cytoplasmic and nuclear volumes of clusters of outer trophoblast and inner pluriblast cells of 3.5-day blastocysts were measured in order to calculate average nucleo-cytoplasmic ratios for each component tissue. Trophoblast measurements were made from whole conceptus images, but pluriblast measurements were made from whole immunosurgically isolated ICM images, since isolation of ICMs improved penetration of nuclear staining at later stages. The mean nucleo-cytoplasmic ratios from each subpopulation differed significantly (Fig. 3e;
$P=0.0015)$. No difference was seen between mural and polar trophoblast subpopulations $(P=0.122)$.

\section{Volumes and nucleo-cytoplasmic ratios from individual cells over the first seven developmental cell cycles}

The averaged data from whole volume analysis suggest continuance of cleavage throughout pre-implantation development. However, the data from the late blastocyst stage indicate that nucleo-cytoplasmic ratios of subpopulations differ. To explore when such differences might emerge, direct measurement of individual blastomere 
(a)

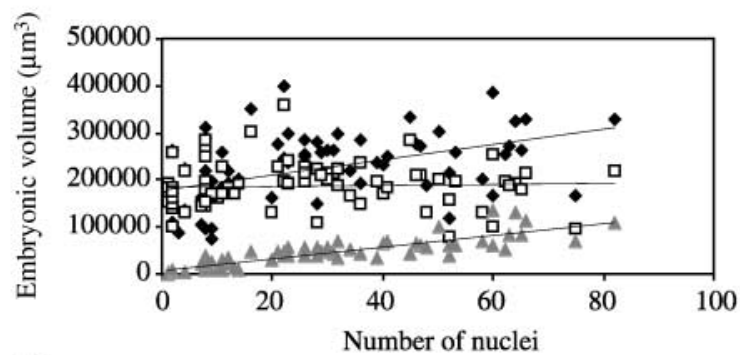

(c)

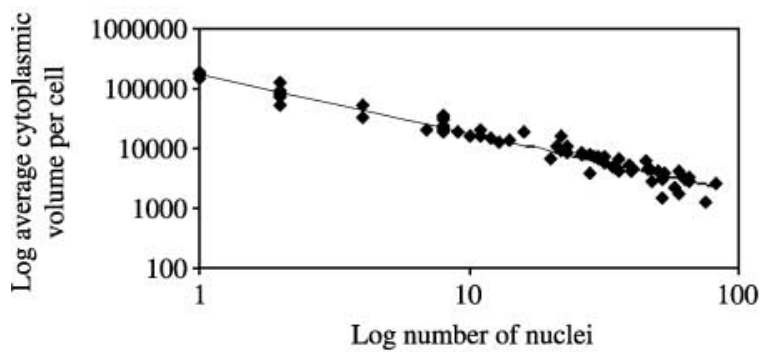

(e)

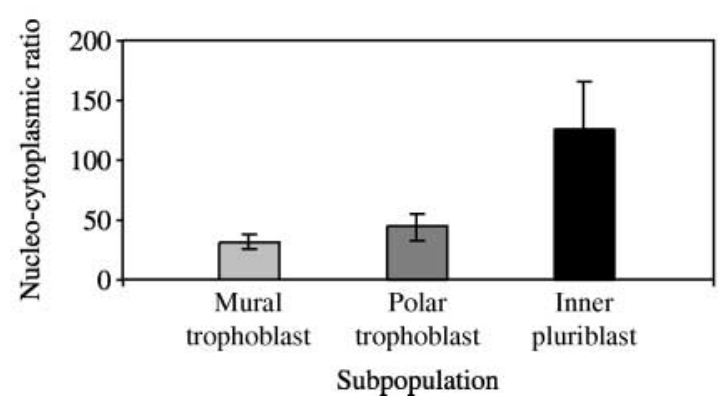

(b)

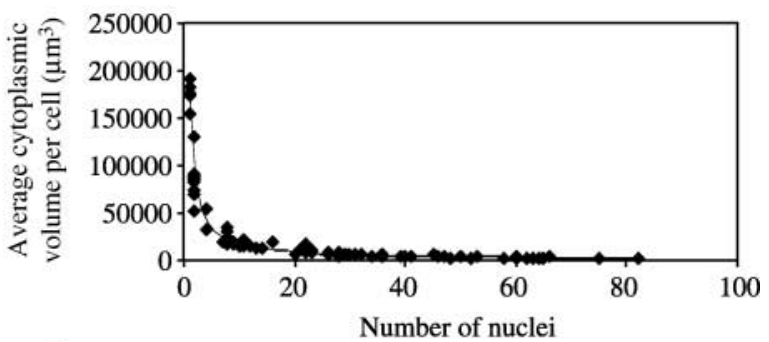

(d)

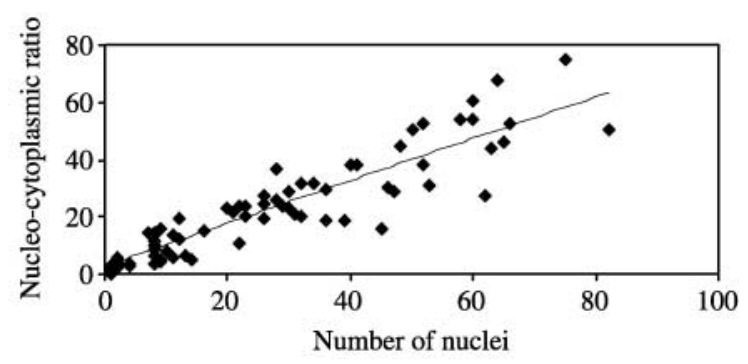

Figure 3 (a) Measured volumes throughout pre-implantation development of whole conceptus $\left(\diamond ; R^{2}=0.2526\right)$, cytoplasmic compartment $(\square$; $R^{2}=0.0012$ ) and nuclear compartment (grey triangles; $R^{2}=0.7951$ ) plotted against total number of nuclei per conceptus. Each point represents a single mouse conceptus ( $n=86$ in panels a-d). (b) Calculated mean cell volume, generated by division of each conceptus volume by the number of nuclei within it. Each point represents a single conceptus. (c) Data from (b) shown as a logarithmic plot; $R^{2}=0.9651$. (d) Calculated mean nucleo-cytoplasmic ratio (shown as a percentage) throughout the pre-implantation period in relation to total cell number. Each point represents a single conceptus and was generated by dividing the summed volume of nuclei by the total cytoplasmic volume; $R^{2}=0.8494$. (e) Nucleo-cytoplasmic ratio (shown as a percentage) from whole subpopulations within 3.5-day blastocysts. ICM data are from isolated ICMs $(n=15)$; trophoblast data are from intact conceptus images $(n=10$ concepti for polar trophoblast and $n=15$ concepti for mural trophoblast). The total cytoplasmic volume of each subpopulation from each embryo was divided by its subpopulation total nuclear volume.

volume and nucleo-cytoplasmic ratio at different stages of development was undertaken. Whole conceptus optical images were taken and individual blastomeres within these selected for cell-by-cell volumetric analysis. Concepti with total numbers approximating a serial doubling of blastomere numbers were used to reduce problems of blastomere asynchrony. Data were collected during the first seven developmental cell cycles and, from the 16-cell stage onwards, cells were categorized according to their inner and outer positions within the conceptus (Table 1). For early cell cycles (first to fourth), distribution plots of cell volumes calculated in this way were found to be normally distributed and unimodal, whereas subsequent cell cycles (fifth to seventh) were spread in an increasingly bimodal distribution. Cell volume was again found to decline by approximately half with each cell cycle (Fig. $4 a)$, and was not significantly different to values calculated from whole conceptus analysis. Converting these data to a linear plot, it is clear that from the fifth (16-cell stage) developmental cell cycle onwards that the volume of outer cells was significantly greater than that of inner cells $(P=0.0131)$. A significant difference was also found in the nucleo-cytoplasmic ratio of inner and outer cells at the late blastocyst stage $(P<0.0001$; Fig. $4 b)$; the nucleocytoplasmic ratio of the trophoblastic population appearing to stabilize. ES cell volume (Fig. 4a) was also measured and found to be higher than that of either inner or outer cells of 3.5-day blastocysts. Within the pluriblast cell population, no difference in volume or nucleo-cytoplasmic ratio of juxta-coelic putative hypoblast precursors and deeper putative epiblast precursors was found (Fig. $4 a$ and b). ES cell nucleo-cytoplasmic ratio (Fig. 4b) was found to be similar to that of trophoblast cells from 3.5day blastocysts. 

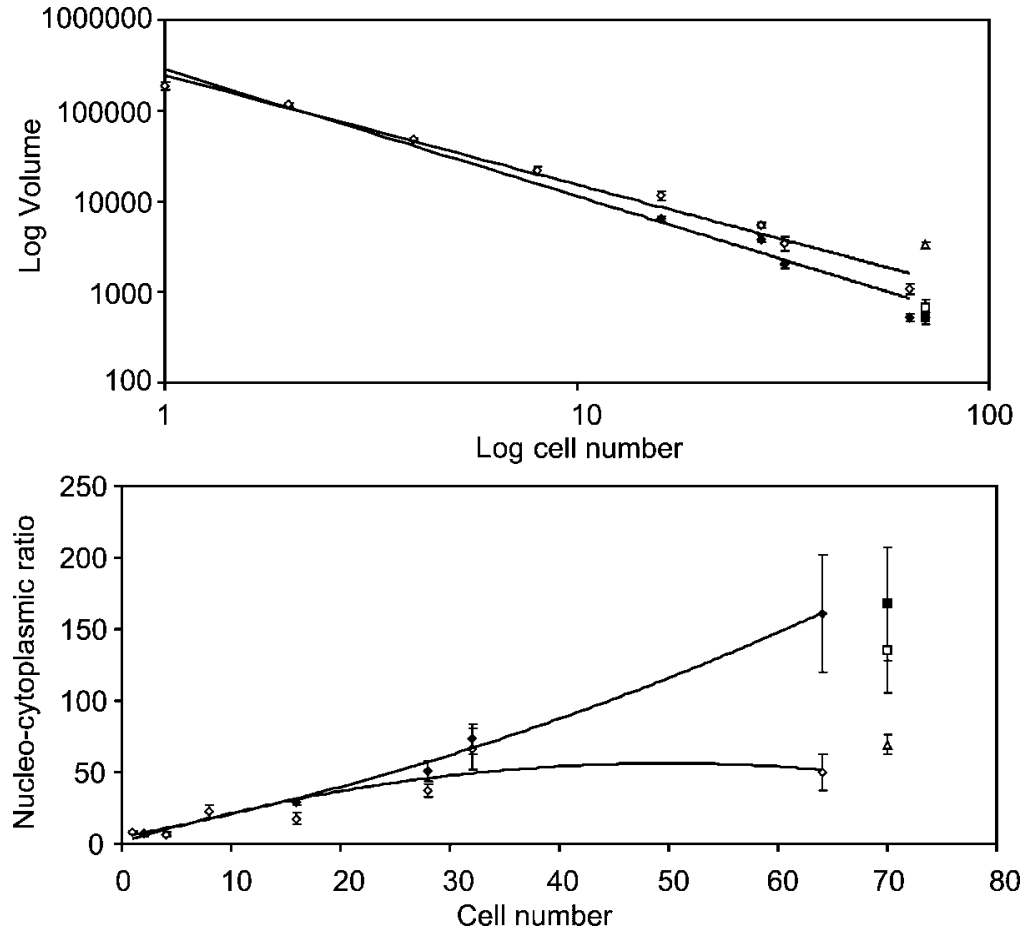

Figure 4 (a) Logarithmic plot of mean values of individual blastomere volumes, separated into inner and outer cells. Cells were designated inner or outer on the basis of examination of optical sections. 95\% confidence intervals are shown; $n$ values for individual blastomeres are shown in Table 1. Note that the data conform to an approximately straight line during the early cell cycles. From the fifth cell cycle (16-cell stage), however, a significant difference in cell volume is shown between inner cells (smaller; $\bullet R^{2}=0.9762$ ) and outer cells (larger; $\diamond ; R^{2}=0.9822$ ). Values for ES cells $(\triangle ; n=40)$, epiblast $(\square ; n=17)$ and hypoblast $(\mathbf{\square} ; n=16)$ are also shown. (b) Mean values of nucleo-cytoplasmic ratio of individual blastomeres; $n$ values for individual blastomeres are shown in Table 1. Pluriblast/inner cells $\left(R^{2}=0.9764\right)$, outer cells $\left(R^{2}=0.8265\right)$. ES cells $(n=40)$, epiblast $(n=17)$, hypoblast $(n=16)$; symbols as in (a).

\section{Discussion}

The total volume of the mouse conceptus remains broadly constant throughout pre-implantation development, but the serial increase in total volume of nuclei leads to an exponential increase in the average nucleo-cytoplasmic ratio. This result suggests that cleavage, as defined by the absence of interphase growth, has not ceased before day 5 and the beginning of attachment. The absence of any net increase in cytoplasmic volume occurs despite the presence of transport systems for the uptake of metabolic substrates (Gardner et al. 2000, Lane \& Gardner 2000) and the machinery to use them for macromolecular synthesis (Epstein 1975, Wales \& Hunter 1990). Previous work (Turner et al. 1992) indicated that embryonic dry mass actually decreased during the pre-implantation period, which is consistent with our findings.

However, the use of averaged whole conceptus data masks variation in emergent subpopulations. The most evident subpopulations to emerge over the pre-implantation period are the trophoblast and pluriblast cell populations that make up the outer transporting epithelium and the ICM of the blastocyst respectively (Johnson \& Selwood 1996, Johnson \& McConnell 2004). Most cells in these two subpopulations are allocated in two waves at the 8- to 16-cell and 16- to 32-cell stages to outer and inner locations respectively (Fleming et al. 1986, Fleming 1987, Johnson \& McConnell 2004). Qualitative observations on 16-cell concepti have shown that, as a population, the inner (pluriblast) cells are smaller than the outer (trophoblast) cells (Handyside 1980, Johnson \& Ziomek 1981). Our quantitative analysis of individual cell volumes has confirmed these earlier observations. Thus, between the one-cell and eight-cell stages, volume distributions are unimodal, and suggest an approximate halving of cell volume with each division. At the 16-cell stage and later, there is a significant difference between the volume of inner and outer cells. By the expanding blastocyst stage, ICM cells continue to reduce in size and so become smaller than those from trophoblastic populations. These volume differences are also reflected in diverging nucleocytoplasmic ratios for trophoblast and pluriblast. Thus, the trophoblast nucleo-cytoplasmic ratio appears to be plateauing (Figs $3 e, 4 a$ and $b$ ). There was no significant volume difference between cells from mural and polar trophoblast subpopulations, nor between ICM cells adjacent to the blastocoele (potential hypoblast?) and those deep within the ICM (potential epiblast?; Fig. 4; Chisholm et al. 1985, Rossant 1986, Johnson \& McConnell 2004).

The size differential between inner and outer cell subpopulations could arise in a number of ways. The differential could reflect an eccentricity of the cleavage planes at the 8- to 16-cell and 16- to 32-cell divisions. Qualitative observations have already suggested that this is the case (Johnson \& Ziomek 1981, Ziomek et al. 1982, Reeve \& Kelly 1983), and it is known that these divisions do generate cell subpopulations that differ in phenotype and potential (reviewed in Johnson \& McConnell 2004). Thus, differential division is likely to provide at least part of the explanation. An alternative explanation might be that a growth increase in cytoplasmic volume occurs during the cell cycle in outer cells, indicating that cleavage ends earlier in this subpopulation. This seems to us unlikely given the overall average stability of cytoplasmic volume and the absence of net total growth, since it would require inner cells to shrink during the cell cycle. 
A third possibility is that at later stages some cells in each of the two subpopulations are in different developmental cell cycles and so of different sizes (assuming that nongrowth cleavage divisions are indeed continuing). This is a possible explanation, given that there is an increasing asynchrony of blastomere divisions with each successive developmental cell cycle, such that cycles might overlap (Chisholm et al. 1985). In addition, there is evidence of differences in cell cycle length between inner and outer cells and of mitotic arrest at later expanded blastocyst stages (Barlow et al. 1972, Johnson \& Ziomek 1981, MacQueen \& Johnson 1983, Surani \& Barton 1984). We have attempted to minimize the problem of overlapping developmental cell cycles by identifying for single-cell analysis concepti with exactly 16, 32 or 64 cells. However, it is possible that trophoblast cells as a population are delayed compared with pluriblast cells and so this may account for part of the size difference between them. A fourth explanation of volume differences between cell subpopulations at blastocyst stages is that from the mid-32-cell stage onwards, blastocoele expansion occurs (Smith \& McLaren 1977, Chisholm et al. 1985). Fluid transport across the mural trophoblast cells (Borland et al. 1977, Wiley 1984) might lead to increases in their volume, although the presence of membrane ion channels, which regulate trophoblastic cell volume and prevent swelling or shrinkage, make this unlikely (Kolajova et al. 2001), as does the observation that there is no significant difference in volume between mural and polar trophoblast (Fig. 3e). Finally, it is possible that the difference in sizes between the two subpopulations might be an artifact of the nonrandom selection of cells for measurement within each subpopulation in the second part of the study. Whereas this cannot be excluded formally, it seems unlikely, since similar differentials were observed when averaged estimates from whole ICM and total trophoblast were made in the first part of the study.

Overall, the most likely interpretation of the subpopulation differentials, given the data on volumetric constancy of the whole conceptus, is that they arise initially by asymmetric division, but developmental cell cycle overlap may account for some of the later divergence. If this conclusion is correct, then cleavage has not ended for either cell subpopulation by the time of attachment. It is reasonable that net growth should not occur until after a secure nutritional contact with the maternal uterine epithelium has been achieved. Interestingly, the volume of the ES cells was measured as being of the same order as day 4-5 trophoblast or pluriblast. ES cells are thought to be equivalent to the late pluriblastic population, so the observation suggests that cleavage may have neared completion by attachment.

Do data in the literature support a nucleo-cytoplasmic ratio of the order seen at the 64-cell stage? Surprisingly, there are few published studies on nucleo-cytoplasmic volumetric ratios in 'mature' cell types. Data derived using a fluorescence partitioning method suggest that a percentage nucleo-cytoplasmic ratio of between 18 and $32 \%$ is found in cultured cells as diverse as endothelial cells, macrophages, fibroblasts, and CV-1 and PtK2 cell lines. Moreover, this ratio range was allometric, being observed in cells of different sizes and with different cDNA contents (Swanson et al. 1991). These values approximate to the values observed around the 40-cell stage and in trophoblast cells and ES cells in culture reported here, but are lower than we observed for pluriblast cells, in which the nucleus comes to occupy a large proportion of the cell. Different measurement techniques may account for part of this difference, but without further work it cannot be excluded that multi-potential stem cells in vivo may pass through a phase of greatly reduced cytoplasmic volume.

There are two implications of our study to which we wish to draw attention. First, a role for nucleo-cytoplasmic ratio as a form of developmental timer has been proposed for a number of developmental systems (reviewed in Johnson \& Day 2000) including the mouse (evidence reviewed in Day et al. 2001). The usual mechanistic model proposed is the titration of a cytoplasmic factor in the oocyte against increasing amounts of DNA (Prioleau et al. 1994). Our observations suggest that such a timing mechanism could (i) operate until attachment and possibly beyond in some or all cells of the conceptus, and (ii) operate differentially in inner and outer cells prior to implantation to activate developmental programmes at different times in the two emergent lineages, perhaps contributing to the initiation of different transcriptional patterns.

Second, the notion of what constitutes cleavage may need to be clarified. We have operated a strict definition based on absence of net growth and the parcelling out of cytoplasm into cells of decreasing size and increasing nucleo-cytoplasmic ratio. A series of other developmental characteristics has been identified in association with cleavage in a range of organisms and this association has sometimes lead to their elision into the definition of cleavage (O'Farrell 2004). For example, in Xenopus, the mid-blastula transition (MBT) occurs during a specific developmental cell cycle and is associated with characteristic changes to cell cycle length, the appearance of $G_{1}$ and $\mathrm{G}_{2}$ phases, cell division asynchrony and motility, changes in protein turnover, and the initiation of transcriptional and apoptotic activity (Newport \& Kirschner 1982a, 1982b, Newport \& Kirschner 1984, Sible et al. 1997, Stack \& Newport 1997). The MBT is often implicitly taken to mark the end of cleavage, even though further reductions in cell volume and nucleo-cytoplasmic ratio may occur. The developmental properties newly expressed at the MBT have also been taken as the mark of a noncleavage phenotype, even though this is clearly not a straightforward case even in Xenopus (Sible et al. 1997). It is certainly not the case in the mouse since transcriptional activation, long and asynchronous cell cycles with checkpoints and $\mathrm{G}$ phases, cell motility and cyto-differentiation are all occurring during, and not after, the period of cleavage. Analogies between different organisms on the basis 
of cleavage similarities and differences may therefore require some qualification (O'Farrell 2004).

\section{Acknowledgements}

We wish to thank Azim Surani, Sheila Barton and Caroline Lee for supply of ES cells, antibodies and media. Part of this work was funded by Wellcome Trust grants to J N S and to M $\mathrm{HJ}$ and $\mathrm{M} \mathrm{H}$ Hastings, MRC Laboratory of Molecular Biology Cambridge. C E M A wishes to thank Trinity College, Cambridge for research studentship funding.

\section{References}

Barlow P, Owen DA \& Graham C 1972 DNA synthesis in the preimplantation mouse embryo. Journal of Embryology and Experimental Morphology 27 431-445.

Bemark M, Khamlichi AA, Davies SL \& Neuberger MS 2000 Disruption of mouse polymerase zeta (Rev3) leads to embryonic lethality and impairs blastocyst development in vitro. Current Biology 10 $1213-1216$.

Borland RM, Biggers JD \& Lechene CP 1977 Studies on the composition and formation of mouse blastocoele fluid using electron probe microanalysis. Developmental Biology 55 1-8.

Chisholm JC 1988 Analysis of the fifth cell cycle of mouse development. Journal of Reproduction and Fertility 84 29-36.

Chisholm JC, Johnson MH, Warren PD, Fleming TP \& Pickering SJ 1985 Developmental variability within and between mouse expanding blastocysts and their ICMs. Journal of Embryology and Experimental Morphology 86 311-336.

Day ML, Winston N, McConnell JL, Cook D \& Johnson MH 2001 tiK $^{+}$toK ${ }^{+}$: an embryonic clock? Reproduction Fertility, and Development 13 69-79.

Epstein CJ 1975 Gene expression and macromolecular synthesis during preimplantation embryonic development. Biology of Reproduction 12 82-105.

Fingar DC, Richardson CJ, Tee AR, Cheatham L, Tsou C \& Blenis J 2004 mTOR controls cell cycle progression through its cell growth effectors S6K1 and 4E-BP1/eukaryotic translation initiation factor 4E. Molecular and Cellular Biology 24 200-216.

Fleming TP 1987 A quantitative analysis of cell allocation to trophectoderm and inner cell mass in the mouse blastocyst. Developmental Biology 119 520-531.

Fleming TP, Cannon PM \& Pickering SJ 1986 The cytoskeleton, endocytosis and cell polarity in the mouse preimplantation embryo. Developmental Biology 113 406-419.

Fulka J Jr, First NL, Fulka J \& Moor RM 1999 Checkpoint control of the G2/M phase transition during the first mitotic cycle in mammalian eggs. Human Reproduction 14 1582-1587.

Gardner DK, Pool TB \& Lane M 2000 Embryo nutrition and energy metabolism and its relationship to embryo growth, differentiation, and viability. Seminars in Reproductive Medicine 18 205-218.

Gunderson H \& Jenson EB 1987 The efficiency of systematic sampling in stereology and its prediction. Microscopy 147 229-263.

Handyside AH 1980 Distribution of antibody- and lectin-binding sites on dissociated blastomeres from mouse morulae: evidence for polarization at compaction. Journal of Embryology and Experimental Morphology 60 99-116.

Howlett SK, Webb M, Maro B \& Johnson MH 1985 Meiosis II, mitosis I and the linking interphase: a study of the cytoskeleton in the fertilised mouse egg. Cytobios 43 295-305.

Johnson MH \& Ziomek CA 1981 The foundation of two distinct cell lineages within the mouse morula. Cell 24 71-80.

Johnson MH \& Selwood L 1996 Nomenclature of early development in mammals. Reproduction, Fertility, and Development 8 759-764.
Johnson M \& Day M 2000 Egg timers: how is developmental time measured in the early vertebrate embryo? Bioessays 22 57-63.

Johnson M \& McConnell JM 2004 Lineage allocation and cell polarity during mouse embryogenesis. Seminars in Cell and Developmental Biology 15 583-597.

Kellogg DR 2003 Wee1-dependent mechanisms required for coordination of cell growth and cell division. Journal of Cell Science $\mathbf{1 1 6}$ 4883-4890.

Kolajova M, Hammer MA, Collins JL \& Baltz JM 2001 Developmentally regulated cell cycle dependence of swelling-activated anion channel activity in the mouse embryo. Development 128 3427-3434.

Lane M \& Gardner DK 2000 Regulation of ionic homeostasis by mammalian embryos. Seminars in Reproductive Medicine $\mathbf{1 8}$ 195-204.

MacQueen HA \& Johnson MH 1983 The fifth cell cycle of the mouse embryo is longer for smaller cells than for larger cells. Journal of Embryology and Experimental Morphology 77 297-308.

Moore GD, Ayabe T, Kopf GS \& Schultz RM 1996 Temporal patterns of gene expression of G1-S cyclins and cdks during the first and second mitotic cell cycles in mouse embryos. Molecular Reproduction and Development 45 264-275.

Newport J \& Kirschner M 1982a A major developmental transition in early Xenopus embryos: II. Control of the onset of transcription. Cell 30 687-696.

Newport J \& Kirschner M 1982b A major developmental transition in early Xenopus embryos: I. characterization and timing of cellular changes at the midblastula stage. Cell 30 675-686.

Newport J \& Kirschner M 1984 Regulation of the cell cycle during early Xenopus development. Cell 37 731-742.

O'Farrell P 2004 Embryonic cleavage cycles: how is a mouse like a fly? Current Biology 14 R35-R45.

Prioleau M-N, Huet J, Sentenac A \& Mechali M 1994 Competition between chromatin and transcription complex assembly regulates gene expression during early development. Cell 77 439-449.

Reeve WJ \& Kelly FP 1983 Nuclear position in the cells of the mouse early embryo. Journal of Embryology and Experimental Morphology 75 117-139.

Roberts N, Cruz-Orive LM, Reid NM, Brodie DA, Bourne M \& Edwards RH 1993 Unbiased estimation of human body composition by the Cavalieri method using magnetic resonance imaging. Journal of Microscopy 171 239-253.

Roberts N, Garden AS, Cruz-Orive LM, Whitehouse GH \& Edwards RH 1994 Estimation of fetal volume by magnetic resonance imaging and stereology. British Journal of Radiology 67 1067-1077.

Rossant J 1986 Development of extraembryonic lineages. In Experimental Appoaches to Mammalian Development, 1st edn. Eds J Rossant \& R Pederson. Cambridge: Cambridge University Press.

Schultz RM 2002 The molecular foundations of the maternal to zygotic transition in the preimplantation embryo. Human Reproduction Update 8 323-331.

Sible J, Anderson J, Lewellyn A \& Maller J 1997 Zygotic transcription is required to block a maternal programme of apoptosis in Xenopus embryos. Developmental Biology 189 335-346.

Smith R \& McLaren A 1977 Factors affecting the time of formation of the mouse blastocoele. Journal of Embryology and Experimental Morphology 41 79-92.

Smith R \& Johnson MH 1986 Analysis of the third and fourth cell cycles of mouse early development. Journal of Reproduction and Fertility 76 393-399.

Solter D \& Knowles BB 1975 Immunosurgery of mouse blastocyst. PNAS 72 5099-5102.

Stack J \& Newport J 1997 Developmentally regulated activation of apoptosis early in Xenopus gastrulation results in cyclin A degration during interphase of the cell cycle. Development 124 3185-3195.

Streffer C, van Beuningen D, Molls M, Zamboglou N \& Schulz S 1980 Kinetics of cell proliferation in the pre-implanted mouse embryo in vivo and in vitro. Cell Tissue Kinetics 13 135-143. 
Surani MA \& Barton SC 1984 Spatial distribution of blastomeres is dependent on cell division order and interactions in mouse morulae. Developmental Biology 102 335-343.

Swanson JA, Lee F \& Knapp PE 1991 Cellular dimensions affecting the nucleocytoplasmic volume ratio. Journal of Cell Biology $\mathbf{1 1 5}$ 941-948.

Takai H, Tominaga K, Motoyama N, Minamishima YA, Nagahama H, Tsukiyama T, Ikeda K, Nakayama K \& Nakanishi M 2000 Aberrant cell cycle checkpoint function and early embryonic death in Chk1(-/-) mice. Genes and Development 14 $1439-1447$.

Telford NA, Hogan A, Franz CR \& Schultz GA 1990 Expression of genes for insulin and insulin-like growth factors and receptors in early postimplantation mouse embryos and embryonal carcinoma cells. Molecular Reproduction and Development 27 $81-92$.

Turner K, Goldstein DJ \& Rogers AW 1992 Variation in the dry mass of mouse embryos throughout the preimplantation period. Human Reproduction 7 112-116.
Waclaw RR \& Chatot CL 2004 Patterns of expression of cyclins A, B1, D, E and cdk 2 in preimplantation mouse embryos. Zygote 12 $19-30$.

Wales RG \& Hunter J 1990 Participation of glucose in the synthesis of glycoproteins in preimplantation mouse embryos. Reproduction, Fertility, and Development 2 35-50.

Wiley LM 1984 Cavitation in the mouse preimplantation embryo: $\mathrm{Na} / \mathrm{K}$-ATPase and the origin of nascent blastocoele fluid. Developmental Biology 105 330-342.

Ziomek CA, Johnson MH \& Handyside AH 1982 The developmental potential of mouse 16-cell blastomeres. Journal of Experimental Zoology 221 345-355.

Received 19 April 2004

First decision 26 May 2004

Revised manuscript received 7 July 2004

Accepted 19 July 2004 